•论坛・

\title{
“2020年后全球生物多样性框架”进展及展望
}

\author{
耿宜佳 田 瑜 李俊生 徐 靖* \\ (中国环境科学研究院国家环境保护区域生态过程与功能评估重点实验室, 北京 100012)
}

\begin{abstract}
摘要: “2020年后全球生物多样性框架”是当前《生物多样性公约》谈判的焦点议题之一。本文阐述了框架制定的 背景, 介绍了“2020年后全球生物多样性框架”不限成员名额工作组(Open Ended Working Group, OEWG), 的谈判过 程, 综合分析了缔约方在各有关碰商进程中的观点, 以及目前缔约方对框架各个要素的共识和分歧, 评估了框架 的制定进展, 并就框架的设计提出四点展望: (1)阐明转型变革的具体实施路径; (2)平衡反映公约三大目标; (3)加 强与其他全球治理进程的协同; (4)强化框架对全球及缔约方履约进展的评估和审查。最后提出对我国的建议: (1) 及时更新国家生物多样性战略与行动计划(National Biodiversity Strategies and Action Plans, NBSAP); (2)加强国内 生物多样性工作的协调; (3)继续加强生态环境执法和责任机制。为缔约方更好参与框架制定进程, 深入了解框架 及其碰商进展提供参考, 并为下一步框架制定提供参考。
\end{abstract}

关键词：可持续发展; 生物多样性公约; 第15次缔约方大会(COP15); 2020年后全球生物多样性框架

\section{Progress and prospects of the Post-2020 Global Biodiversity Framework}

\begin{abstract}
Yijia Geng, Yu Tian, Junsheng Li, Jing Xu*
State Environment Protection Key Laboratory of Regional Eco-process and Function Assessment; Chinese Research
\end{abstract} Academy of Environmental Sciences, Beijing 100012

\begin{abstract}
The Post-2020 Global Biodiversity Framework is one of the main focuses of current negotiations for the Convention on Biological Diversity. This paper outlines the background of the "Framework," introduces the OEWG-1 negotiation process, comprehensively analyzes the views of the Parties in the relevant consultation processes, introduces the current consensuses and differences of the Parties on the various elements of the "Framework," assesses the progress of the "Framework," and puts forward four suggestions on the design of the "Framework". These suggestions include: (1) Clarify the specific implementation path of transformational change; (2) Balance the three major objectives of the CBD; (3) Strengthen collaboration with other global governance processes; (4) Strengthen the "Framework" assessment and review of global and the Parties' progress in compliance. Finally, we put forward suggestions for China: (1) Update the domestic NBSAP in a timely manner; (2) Strengthen the coordination of domestic biodiversity work; (3) Continue to strengthen the ecological environmental law enforcement and responsibility mechanism. These recommendations provide a reference for the Parties to better participate in the "Framework" development process and gain insight into the framework and its progress in order to provide useful feedback for the formulation of the "Framework."
\end{abstract}

Key words: sustainable development; Convention on Biological Diversity; COP15; Post-2020 Global

2020年是《2011-2020年生物多样性战略计划》 及其20个爱知目标实施的收官之年, 但全球生物多 样性保护形势依然严峻(薛达元, 2017; 徐靖等, 2018)。根据生物多样性和生态系统服务政府间科学 政策平台(IPBES)发布的生物多样性和生态系统服
务全球评估报告, 爱知目标中, 仅有少数有望实现 或取得积极进展, 大多数进展有限, 甚至偏离目标 (IPBES, 2019)。2020年将达成的“2020年后全球生物 多样性框架” (以下简称框架)是未来全球生物多样 性进程的纲领性文件, 将指导未来十年全球生物多

收稿日期: 2019-11-20; 接受日期: 2019-12-18

基金项目: 生态环境部生物多样性保护专项(22110404001)

* 通讯作者 Author for correspondence. E-mail: xujing263@163.com 
样性各国行动, 促进2050愿景, 因此广受各方关 注。框架将是《生物多样性公约》(以下简称《公约》) 第十五次缔约方大会(以下简称COP15)的核心成果, 具有里程碑意义。我国是COP15东道国, 需要促进 各方对框架关键内容达成共识。本文分析了各方对 框架的范围与要素的主要观点与分歧, 对“2020年 后全球生物多样性框架” 不限成员名额工作组 (Open Ended Working Group, OEWG)第一次会议 (OEWG-1)通过的框架提纲进行了分析, 对框架后 续磋商进程进行展望并提出了建议, 以期为我国深 入参与框架的制定进程提供参考。

\section{1 背景}

框架的制定是《公约》多年期工作方案的决定 事项。2014年，《公约》第十二次缔约方大会通过 了将在COP15期间审议框架的决定 ${ }^{1}$ 。2016年, 《公 约》第十三次缔约方大会要求秘书处编制框架筹备 进程及时间表 ${ }^{2}$ 。2017年，《公约》秘书处在广泛征 求缔约方及其他利益做关方意见的基础上，起草了 《关于编制框架的全面和参与性进程的建议》(简称 《建议》)。2018年，《公约》第十四次缔约方大会 在《建议》的基础上, 重点讨论了编制框架的工作 安排，包括机构设置、时间安排及框架推进方式等。 会议决定组建OEWG专门负责制定框架。OEWG与 《公约》两个附属机构加强议题协调, 以互相支持 的方式开展工作, 计划COP15之前至少召开两次会 议，必要时可视情况额外召开会议 ${ }^{3}$ 。

2019年2月起, 《公约》秘书处在全球五大区域 先后组织了区域磋商会议, 各方已就框架讨论多次, 24 个缔约方和 120 个观察员先后提交了关于框架的 书面意见 ${ }^{\oplus}$ 。2019年8月27-30日, OEWG-1在肯尼亚 内罗毕召开。会议通过了与《公约》其他机构和进 程相衔接的初步任务清单及供进一步讨论的可能 要素 ${ }^{(5)}$

\section{OEWG-1谈判过程}

OEWG-1关于框架结构和范围的可能要素的非 正式文件将框架的要素归为以结果为导向的要素 (愿景、任务、目标和指标), 有利于执行的条件和手 段, 规划和问责的模式、机制和工具以及跨领域办 法和问题四类 ${ }^{6}$ 。四大类别共同组成了框架的主要 结构, 彼此区别又相互联系。各方对该归类方法基
本表示认可, 并按此分类阐述了对框架要素的意见 (Peter et al, 2019)。以此为基础, OEWG共同主席成 立了讨论组, 就框架要素的意见进行了讨论。

目前，第一份包括框架范围和结构提纲的文件 包括8项内容：(1)框架制定的理由和范围，提纲彗 领地叙述框架编制的理由，明确涉及范围; (2) 2050 愿景，描述框架至2050年的远期目标；(3) 2030年任 务或顶级目标和阶段性战略目标，以适当的方式 为框架确定一个近期目标；(4)目标和指标，根据全 球生物多样性状况、受威胁因素和治理情况, 确定 框架的优先领域, 明确需要达到的具体目标和评估 指标; (5)执行手段和促进条件, 明确加强框架执行 的各种手段和条件; (6)跨领域问题及实现途径，在 实现框架各项目标时都需要考虑的问题和解决方 法; (7)透明的执行、监测和报告机制，提高框架执 行效果的机制、工具和方法; (8)对外传播和联络, 宣传和介绍框架，引起外界对框架的关注和重视的 手段和方法。

该文件是具有一定共识基础的框架提纲，能够 较好地覆盖框架的可能要素, 广泛满足各方关切, 是OEWG共同主席起草框架文本的重要参考 ${ }^{\circledR}$ 。虽 然未来各方仍可对该文件内容进行修改完善，但在 很大程度上将以该文件为基础展开谈判。OEWG共 同主席和秘书处将在缔约方大会主席团监督下，于 2019年11月前形成框架范围和结构草稿，并在2020 年2月OEWG第二次会议前形成框架碰商案文。

\section{3 各方观点及分歧}

\section{1 关于框架理由和范围}

是否通过援引转型变革解决生物多样性问题 是起草框架理由和范围的主要分歧点。转型变革是 指社会、人口、经济等政策和行动缓解生物多样性 丧失的间接驱动力(IPBES, 2019)。欧盟等对在该部 分援引转型变革理论态度积极, 认为全球生物多样 性治理需要新理论指导, 从行动层面促使全球生物 多样性治理行动转变; 巴西、阿根廷和非洲集团 ${ }^{(8)}$
(1) $\mathrm{UNEP} / \mathrm{CBD} / \mathrm{COP} / 12 / \mathrm{DEC} / 31$
(2) $\mathrm{UNEP} / \mathrm{CBD} / \mathrm{COP} / 13 / \mathrm{DEC} / 1$
(3) $\mathrm{UNEP} / \mathrm{CBD} / \mathrm{COP} / \mathrm{DEC} / 14 / 34$
(4) $\mathrm{CBD} / \mathrm{WG} 2020 / 1 / 2$
(5) https://www.cbd.int/meetings/WG2020-01
(6) UNEP/CBD/WG2020/1/3
(7) $\mathrm{CBD} / \mathrm{WG} 2020 / 1 / \mathrm{L} .2$
(8) https://www.cbd.int/conferences/post2020/submissions/2019-008 
等则认为, 转型变革理论还不成熟, 其概念和内涵 还不清晰, 其涉及的行动能够在多大程度上有助于 加强全球生物多样性治理也缺乏适当的评估, 因此 难以在框架中予以体现。由于IPBES全球评估报告 和其他一些有关生物多样性的评估报告已经发布, 对于生物多样性和生态系统服务的重要性、生物多 样性现状及其对人类福祉的影响、应对生物多样性 挑战的必要性、导致生物多样性丧失的直接和间接 驱动因素、履约面临的挑战等内容各方基本达成 一致。

\subsection{0 愿景}

是否需要进一步解释2050愿景以指导框架的 起草和制定是OEWG-1的另一个主要分歧点。《公 约》缔约方大会已决定将2050愿景作为框架的一部 分 ${ }^{1}$ 。2050愿景的内容将会指导框架其他内容的起 草和制定。讨论中, 一些缔约方认为, 应当进一步 解释2050愿景以及如何通过转型变革实现这一愿 景, 使其更好地为框架其他内容的制定提供参考, 但也有缔约方认为 2050 愿景的表述已十分清晰, 不 必进行更多解释。

\subsection{0 年任务或顶级目标和阶段性战略目标}

缔约方对框架具体任务的结构和内容的分歧 也较大。欧盟、瑞士 等主张建立一个以2030、2040 为任务节点, 2050为最高目标的任务体系, 并建议 以物种灭绝速率作为衡量任务实现情况的关键指 标。他们认为, 物种丧失对于公众十分直观, 易于 理解, 能够调动公众采取行动应对生物多样性挑战, 而通过物种灭绝速率也能够实现对框架执行进展 的评估及监督。非洲、巴西等则倾向于制定一个有 明确时间节点的 2030年任务, 该任务可描述为至 2030年生物多样性的状态，也可描述为实现《公约》 三大目标需要采取的行动。巴西等还强调此类行动 不应包含可持续的生产和消费，并应当与联合国可 持续发展目标相关联。

\section{4 关于目标和指标}

缔约方对框架目标的结构基本持一致意见, 认 为目标应当有层次, 设置少量战略目标, 其下设置 一定数量的具体目标, 具体目标下再设置更多更加 具体的次级目标, 并建立指标体系以便评估。缔约 方对战略目标的范围分歧较大。欧盟等主张将解决 生物多样性丧失的直接和间接驱动力作为框架的 目标。直接驱动力包括土地和海洋用途的变化、生
物的直接利用、气候变化、污染、外来物种入侵; 间 接驱动力包括生产和消费模式、人口、动态和趋势、 贸易、技术创新、地方和全球治理(IPBES, 2019)。 巴西、阿根廷等国表示, 框架具体目标的设定应遵 循公约三大目标, 平衡考虑保护、可持续利用和惠 益分享。讨论中，巴西、阿根廷等国十分关注可持 续相关目标的设计, 多次强调框架目标应与联合国 可持续发展目标保持协调一致，建议专门就可持续 利用生物多样性的具体目标进行讨论，以便尽早形 成共识。非洲集团则主张将资源调动、能力建设等 保障框架执行的条件作为框架的目标之一，认为此 举将有助于框架执行过程中获取更多资源和协助。

具体目标的设计原则也是讨论的重点。《20112020生物多样性战略计划》目标难以实现的原因包 括: (1)目标具体表述不清(Polak et al, 2015; Wood et al, 2015; Juffe-Bignoli et al, 2016); (2)可量化程度不 高(Butchart et al, 2016; Juffe-Bignoli et al, 2016; Watson et al, 2016); (3)缺少合适的评估指标(Jorgensen, 2013); (4)目标力度不足(Harris et al, 2014)。此 外, 国家战略对 《2011-2020生物多样性战略计划》 目标缺乏响应也是原因之一。针对这种情况，欧盟 提出目标应该具体(specific)、可测量(measurable)、 可实现(achievable)、基于成果(result-based)和有时 限(time-bound)。该观点被许多缔约方所认同，其他 缔约方尚未提出更有说服力的观点。瑞士等则强调 与其他多边环境协定和进程的协同一致, 认为具体 目标既要反映其他多边环境协定的工作进展，又不 能简单重复其他进程已经设置的目标。

对于评估指标, 缔约方具有较多共识, 并提出 了指标的可能来源: 缔约方大会确定的指标 ${ }^{3} 、 2030$ 联合国可持续发展议程、IPBES评估指标及生物多 样性伙伴关系等其他进程已经确定的指标，提出指 标和基线应与目标同时设定，并在框架通过后对指 标和基线进行审查以便改进。

\section{5 关于实施手段和保障条件}

调动资源、健全资金机制、开展能力建设、加 强科技合作与技术转让, 以及推动信息管理与共享 是确保框架实施的有效手段, 也是实现公约三大目 标的基础性保障条件, 发展中国家缔约方对此有较

\footnotetext{
(1) UNEP/CBD/COP/14/DEC/3

(2) https://www.cbd.int/conferences/post2020/submissions

(3) $\mathrm{UNEP} / \mathrm{CBD} / \mathrm{COP} / 13 / \mathrm{DEC} / 28$
} 
大需求。保护和可持续利用土著人民和地方社区持 有的传统知识也有利于框架的有效实施, 缔约方对 将以上要素纳入框架没有分歧。

自然资本核算和环境治理是在该问题上的主 要分歧。将生态系统服务作为一种自然资本是近年 来国际上生物多样性领域的热点之一。欧盟及一些 国际组织认为自然资本核算是创新性融资机制的 一部分, 有利于从更多渠道进行融资。玻利维亚等 安第斯共同体国家认为生态系统服务难以使用货 币价值进行衡量。一些缔约方认为环境治理概念和 内涵过于宽泛, 需要明确哪些环境治理措施能够有 助于框架的实施。

\section{6 关于执行、监测和报告机制}

加强国家生物多样性战略与行动计划和国家 报告是此次会议的主要共识，可能涉及的内容包括： 加强对国家生物多样性战略与行动计划的指导, 提 高国家生物多样性战略与行动计划和国家报告的 质量和可比性等。但在执行机制和手段方面, 缔约 方分歧较大。一些缔约方认为需要讨论澄清“自愿 承诺”这一概念。加拿大等认为“自愿承诺” 属于实施 手段, 可以放在执行手段部分, 这意味着承诺的范 围限于为框架的实施提供各种条件。此外, 德国等 认为, 缔约方在任何情况下的承诺都是强制性的, “自愿承诺”只能由非国家行为体做出, 其原因是如 果允许缔约方“自愿承诺”将动摇国家生物多样性战 略与行动计划作为国家承诺载体的基础。一些缔约 方反对用“遵约”来表述该机制，认为框架是为实现 公约三大目标制定的战略性文件, 不存在为框架建 立遵约机制的法律基础。“棘轮机制”(ratchet mechanism) 是《巴黎协定》下建立的强化执行机制, 部分非政府组织积极推动使用这一机制。一些缔约 方认为, 目前该机制并未在《巴黎协定》进程中 真正执行, 其在框架内的可操作性和适用性有待 商榷。

\section{7 其他问题}

缔约方在跨领域问题及外联交流、认识和理解 两大问题上分歧较大。一些缔约方认为文件所列的 跨领域问题可在目标等部分得到妥善解决, 但一些 缔约方则担心这种做法将使跨领域问题受到忽视。 对于外联、认识和理解, 许多缔约方认为, 此项工 作需与框架制定进程同时进行, 作为框架要素纳入 并在其通过后再行实施。

\section{展望与建议}

\section{1 展望}

OEWG-1是一次由缔约方主导的、具有广泛包 容性的会议。会议讨论形成的框架提纲全面反映了 各方的观点和意见，最大范围地明确了框架范围和 各主要要素, 为进一步凝聚缔约方共识提供了良好 的基础。通过此次会议，缔约方均意识到框架的目 标要素并非框架的全部内容, 还需就框架的执行手 段, 保障措施和监测、评价和报告等方面进行全面 统筹考虑, 今后缔约方的提案基本不会超出这一范 畴, 这为缔约方进一步思考框架各要素具体内容提 供了共同遵循的基础。框架提纲也在一定程度上对 OEWG共同主席起草框架提供了支持，在起草框架 文本时，需要考虑这一具有广泛共识的提纲。基于 此, 本文就框架的设计提出以下建议, 为缔约方更 好参与框架制定进程提供参考:

(1)在框架顶层目标设计时阐明转型变革的实 施路径。顶层目标指框架在2050年需要达到的长期 目标和2030任务。《2011-2020生物多样性战略计 划》包含了与自然和谐共生的2050愿景，但未说明 通过何种路径实现这一愿景。IPBES提出了转型变 革，但仅强调其意味着对各种技术、经济和社会因 素进行系统性、根本性重组, 并未列举在生物多样 性全球治理领域需要采取哪些转型变革措施。框架 应当在梳理总结各国环境治理经验的基础上，对转 型变革的范围、内涵、具体措施和行动等进行更加 清晰的解释, 才能使缔约方就如何实现转型变革形 成共识, 为框架战略目标和具体目标的设计提供 指导。

(2)制定平衡反映公约三大目标的战略目标。强 调生物多样性保护, 对其他两个目标重视不足是缔 约方《2011-2020生物多样性战略计划》执行中的 问题。目前，欧盟等发达国家缔约方主要关注可持 续生物多样性相关目标，非洲等发展中国家缔约方 则十分关注利用遗传资源产生惠益的分享问题，包 括如何解决遗传资源数字序列信息(DSI)的利用和 惠益分享。此外，退化生态系统的恢复也受到非洲 国家广泛关注，健康的生态系统能够在减缓气候变 化，改善当地生态环境质量，提高当地社区人民生 活水平等方面发挥多重作用。作为实现公约目标的 战略文件, 框架需要针对公约的三大目标相对平衡 
地提出具体任务和措施，可以考虑将保护、可持续 利用和惠益分享同时作为框架的战略目标, 将改善 和恢复生态系统作为惠益分享的一部分, 通过在战 略目标下各项行动的实施，促进三大目标的实现。

(3)框架具体目标要考虑与其他全球治理进程 的协同。土地和海洋用途的变化、生物的直接利用、 气候变化、污染和外来物种入侵是导致生物多样性 丧失最重要的直接驱动因素, 直接驱动因素又受生 产和消费模式、人口动态和趋势、贸易、技术创新 及环境治理等间接驱动因素影响, 这些间接驱动因 素受社会价值观和行为影响。框架的具体目标需要 重点解决以上直接因素对生物多样性的影响, 也需 要与其他全球治理进程加强协调。可行的做法是将 控制或降低直接因素作为具体目标, 同时在识别直 接和间接驱动因素之间联系的基础上，设置评估间 接驱动因素的指标, 通过借鉴其他治理进程的数据 和信息, 使框架能够全面反映生物多样性相关治理 活动的成效。

(4)强化框架对全球及缔约方履约进展的评估 和审查。《2011-2020生物多样性战略计划》的经验 教训之一是缺乏对缔约方履约进展的科学有效评 估。公约现有的评估和审查工具包括国家报告、自 愿性同行评议和《全球生物多样性展望》等，可以 考虑采取规范国家报告格式, 明确报告内容, 协同 公约及其议定书报告周期, 以及第三方评估等多种 形式提高国家报告质量, 为后续监督和审查提供良 好基础。

\section{2 对我国的建议}

(1)及时更新国家生物多样性战略与行动计划 (NBSAP)。框架中确定的新目标将替代爱知生物多 样性目标, 指明未来全球生物多样性保护与可持续 利用的方向。作为实施框架的基础工具, 缔约方需 要通过更新NBSAP, 将全球目标转化为本国履约目 标。建议我国根据新设立的框架目标及时修订国内 NBSAP, 明确提出国家履约目标, 为未来组织开展 全国生物多样性保护和可持续利用工作指明方向。 新的国家目标应当与框架目标有明确的衔接关系, 确保我国的履约成绩能够在评估框架执行进展时 得到更好的体现和反映。

(2)加强国内生物多样性工作的协调。作为全球 生物多样性保护的框架性公约, 框架中的许多目标 可能需要除环境部门以外的其他部门配合执行, 只
有这样才能解决导致生物多样性丧失的间接驱动 力, 促进《生物多样性公约》三大目标的实现, 特 别是可持续利用生物多样性目标的实现。生物多样 性的主流化是确保NBSAP有效执行的有效手段, 需要采取强有力的政府行动, 建立健全相关部门的 生物多样性管理机构和地方政府生物多样性管理 协调机制。我国已经建立的中国生物多样性保护国 家委员会可以在这方面进一步发挥协调作用，推进 NBSAP在国内的实施。

(3)继续加强生态环境执法和责任机制。健全的 责任机制能够有效提升缔约方的履约成效，促进框 架的实施，若不加强问责，框架的实施就有可能重 蹈《2011-2020生物多样性战略计划》实施不力的 覆辙。用最严格制度最严密法治保护生态环境是我 国推进生态文明建设的重要原则, 未来需要通过健 全和完善生物多样性相关法律体系，加强相应的执 法力度, 确保NBSAP的各项目标切实有效履行。

\section{参考文献}

Butchart SHM, Marco MD, Watson JEM (2016) Formulating smart commitments on biodiversity: Lessons from the Aichi Targets. Conservation Letters, 9, 457-468.

Harris L, Nel R, Stephen H, Kerry S, David S (2014) Setting conservation targets for sandy beach ecosystems. Estuarine Coastal \& Shelf Science, 150, 45-57.

IPBES (Intergovernmental Science-Policy Platform on Biodiversity and Ecosystem Services) (2019) Summary for Policymakers of the Global Assessment Report on Biodiversity and Ecosystem Services of the Intergovernmental Science-Policy Platform on Biodiversity and Ecosystem Services. https://www.ipbes.net/system/tdf/ipbes _7_10_add-1-_advance.pdf?file=1\&type=node\&id=35329. (accessed on 2019-07-08)

Jorgensen (2013) Ecological restoration in the Convention on Biological Diversity targets. Biodiversity \& Conservation, 22, 2977-2982.

Juffe-Bignoli D, Harrison I, Butchart SH, Flitcroft R, Hermoso V, Jonas H, Lukasiewicz A, Michele T, Eren T, Heather B (2016) Achieving Aichi Biodiversity Target 11 to improve the performance of protected areas and conserve freshwater biodiversity: Elements needed to meet a global target for protected areas. Aquatic Conservation Marine \& Freshwater Ecosystems, 26, 133-151.

Peter D, Laura BC, Natalie J (2019) Summary of the first substantive session of the Ad Hoc Open Ended Working Group towards a Global Pact for the environment: 14-18 January 2019. Earth Negotiations Bulletin, 35(1), 1-14.

Polak T, Watson JE, Fuller RA, Joseph LN, Martin TG, 
Possingham HP, Venter O, Carwardine J (2015) Efficient expansion of global protected areas requires simultaneous planning for species and ecosystems. Royal Society Open Science, 2, 150107.

Watson JE, Darling ES, Venter O, Maron M, Walston J, Possingham HP, Dudley N, Hockings M, Barnes M, Brooks TM (2016) Bolder science needed now for protected areas. Conservation Biology, 30, 243-248.

Wood G, Whyatt D, Stevens C (2015) Towards the integration of urban planning and biodiversity conservation through collaboration. Environmental Technology \& Innovation, 4, 218-226.
Xu J, Geng YJ, Yin SL, Shi CZ (2018) A study on elements of Post-2020 Global Biodiversity Framework eased on sustainable development goals, 46(23), 17-22. (in Chinese with English abstract) [徐靖, 耿宜佳, 银森录, 史朝中 (2018) 基于可持续发展目标的“2020年后全球生物多样 性框架”要素研究. 环境保护, 46(23), 17-22.]

Xue DY (2017) The latest development for implementation of the Convention on Biological Diversity. Biodiversity Science, 25, 1145-1146. (in Chinese) [薛达元 (2017) 《生 物多样性公约》履约新进展. 生物多样性, 25, 1145-1146.]

(责任编委：马克平 责任编辑：时意专) 